\title{
Comparison of Periodic Flow Fields in a Radial Pump among CFD, PIV, and LDV Results
}

\author{
Jianjun Feng, F.-K. Benra, and H. J. Dohmen \\ Department of Mechanical Engineering, Faculty of Engineering, University of Duisburg-Essen, 47048 Duisburg, Germany \\ Correspondence should be addressed to Jianjun Feng, jianjun.feng@uni-due.de
}

Received 11 March 2009; Accepted 1 August 2009

Recommended by Gerard Bois

\begin{abstract}
The interaction between the impeller and the diffuser is considered to have a strong influence on the unsteady flow in radial pumps. In this paper, the unsteady flow in a low specific speed radial diffuser pump has been simulated by the CFD code CFX-10. Both Particle Image Velocimetry (PIV) and Laser Doppler Velocimetry (LDV) measurements have been conducted to validate the CFD results. Both the phase-averaged velocity fields and the turbulence fields obtained from different methods are presented and compared, in order to enhance the understanding of the unsteady flow caused by the relative motion between the rotating impeller and the stationary diffuser. The comparison of the results shows that PIV and LDV give nearly the same phase-averaged velocity fields, but LDV predicts the turbulence much clearer and better than PIV. CFD underestimates the turbulence level in the whole region compared with PIV and LDV but gives the same trend.
\end{abstract}

Copyright ( $\odot 2009$ Jianjun Feng et al. This is an open access article distributed under the Creative Commons Attribution License, which permits unrestricted use, distribution, and reproduction in any medium, provided the original work is properly cited.

\section{Introduction}

The internal flow developing in a radial diffuser pump is extremely complicated and highly turbulent, caused by streamline curvatures, system rotation, flow separations, rotor-stator interaction, and turbulence effects. The rotorstator interaction is assumed to have an important influence on the time-variant flow behavior in the case of a small radial gap between the impeller trailing edge and the diffuser leading edge [1].

With the development of computational algorithms and computer technology, (CFD) Computational Fluid Dynamics has become a power tool to calculate the unsteady flow in radial pumps [2-7]. Particle Image Velocimetry (PIV) is a noncontact measurement technique for obtaining instantaneous velocity field, in which the measured property is the distance travelled by seeding particles in the flow within a known time interval [8]. PIV has the advantage of measuring the whole flow field instantaneously, whether it is steady or unsteady. PIV has been widely applied in the flow measurements in radial pumps. Akin and Rockwell [9] applied PIV to study the impeller wake and its interaction with diffuser vanes. Sinha and Katz [10] used PIV results to identify the unsteady flow structure and turbulence in a radial pump. More work by PIV measurements can be found by Wuibaut et al. [11, 12] and Feng et al. [13, 14]. Compared with PIV, Laser Doppler Velocimetry (LDV) is more time-consuming but gives more accurate results due to the measurement directly on the points of interest. LDV measurements conducted in radial pumps have been reported by Akhras et al. [15], Hajem et al. [16], Pintrand et al. [17], and Akhras et al. [18].

In this paper, the unsteady flow field in a low specific speed $\left(n_{s}=22.6\right)$ radial diffuser pump has been examined in detail both numerically by the CFD code CFX-10 and experimentally by PIV and LDV. The velocity and turbulence fields obtained from different methods are compared both qualitatively and quantitatively at the design operating point. This can enhance the comprehension of the unsteady phenomena during the conveyance of the fluid from the impeller to the stator due to the change of frame of reference.

\section{Numerical and Experimental Setups}

2.1. Geometry of the Pump. Figure 1 shows a view of the pump test stand. The specific speed of the pump is $n_{s}=22.6$. 


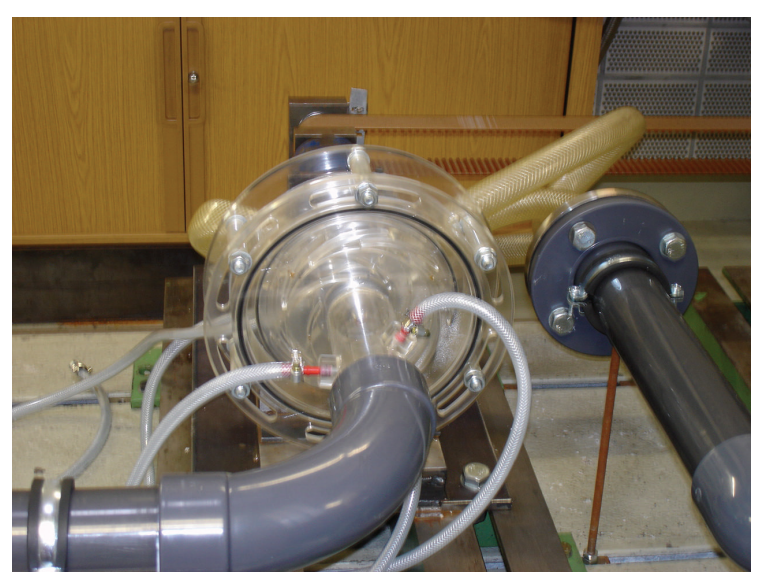

FIgURE 1: View of the pump test stand.

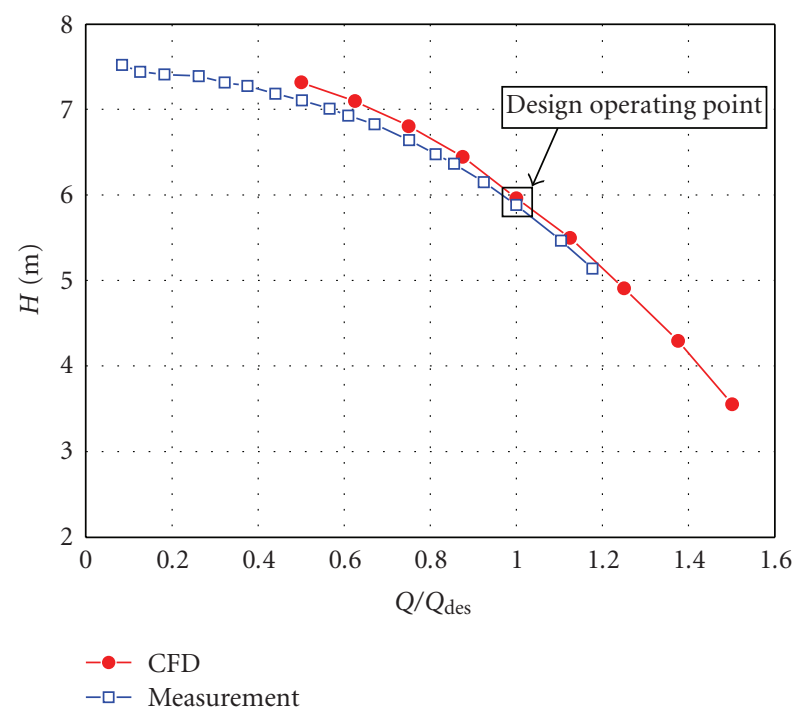

Figure 2: Characteristic curve.

The impeller has six two-dimensional and strongly backswept blades. The radial gap between the impeller trailing edge and the diffuser leading edge is 3\% of the impeller outlet radius. The axial gap between the impeller shroud wall and the casing is $1.85 \mathrm{~mm}$, accounting for $14.6 \%$ of the impeller blade height. Both the diffuser and return channel have nine two-dimensional vanes. The specifications of the pump stage are illustrated in Table 1. In addition, the head curves are compared in Figure 2 between CFD calculations and measurements.

2.2. CFD Simulation. Three-dimensional, unsteady Reynolds-averaged Navier-Stokes equations are solved by the CFD code CFX-10. The structured grids for the computational domains are generated by using the commercial software ICEM-CFD 10. The impeller side chambers are also included in the grids to take leakage flow effects into account. The turbulence is simulated by the SST (shear stress transport) turbulence model [19]. The interface between the impeller and the diffuser is set to "transient

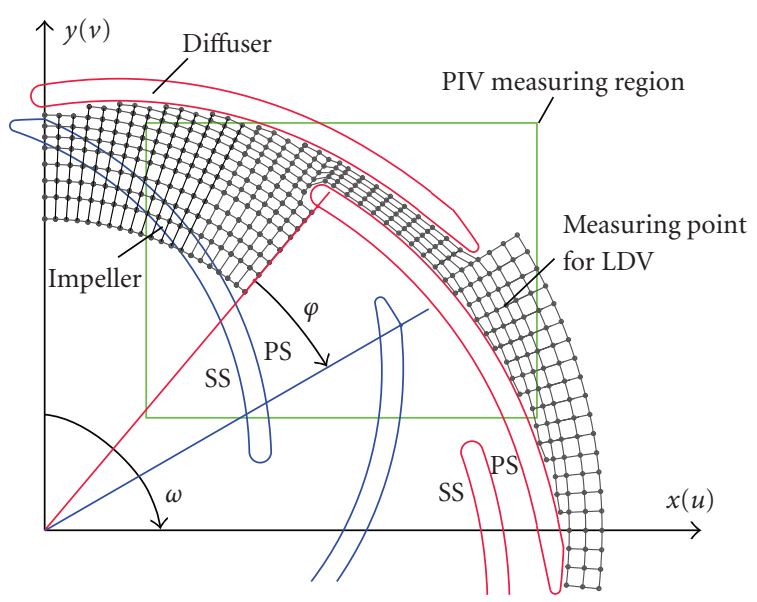

FIgURE 3: PIV and LDV measuring regions.

rotor-stator," in which the relative position between the rotor and the stator is updated each time step. The computational grids and boundary conditions can be taken from our previous work [20].

2.3. PIV and LDV Measurements. The measuring region for the PIV measurement is indicated by a green rectangle in Figure 3, with the size of $72.5 \mathrm{~mm} \times 58 \mathrm{~mm}$. The light source for the PIV measurements is a double-cavity $532 \mathrm{~nm} \mathrm{Nd}$ YAG laser with a repetition rate of $15 \mathrm{~Hz}$ and the energy of $120 \mathrm{~mJ} / \mathrm{pulse}$. The water is seeded with polyamide particles with an average diameter of $20 \mu \mathrm{m}$ and a density of 1.02 relative to water. The images are recorded by a $1024 \times 1280$ pixels CCD camera. An encoder installed on the pump shaft is used to synchronize the measurement with the relative impeller position. At each relative impeller position, 200 double-frame images are recorded.

For the LDV measurement, the light source is an ArgonIon laser with a maximum power of $5 \mathrm{~W}$ operating in multiline mode. The multicolor beam separator is utilized for obtaining the green $(514.5 \mathrm{~nm})$ and blue $(488 \mathrm{~nm})$ beams. An optical probe with a $500 \mathrm{~mm}$ focusing lens is used to derive a two-pair beam configuration. The optical probe with the lens is mounted on a two-axis traversing system in order to place the probe volume at the location of interest. The measuring points are indicated by black dots in Figure 3. The measuring region covers the rear part of the impeller, starting from $r / R_{2}=0.757$ due to the design limitation of the pump test stand, and a full diffuser channel. The encoder for the PIV measurement is used here to relate the measurement to the angular positions of the impeller. For each measuring point in the impeller, 100000 sets of data are acquired, and 50000 sets of data are collected for each measuring point in the diffuser.

2.4. Data Postprocessing for PIV and LDV. During the measurements, an instantaneous velocity component consists of a phase-averaged part $(\bar{u}(x, y, \varphi)$ or $\bar{v}(x, y, \varphi))$ and a random one $\left(u_{i}^{\prime}(x, y, \varphi)\right.$ or $\left.v_{i}^{\prime}(x, y, \varphi)\right)$, as denoted in (1). The 
TABLe 1: Specifications of the pump stage.

\begin{tabular}{|c|c|c|c|c|c|}
\hline \multicolumn{3}{|c|}{ Impeller } & \multicolumn{3}{|c|}{ Return channel } \\
\hline Number of blades & $Z_{i}$ & 6 & number of vanes & $Z_{r}$ & 9 \\
\hline Inlet radius & $R_{1}$ & $40 \mathrm{~mm}$ & inlet radius & $R_{5}$ & $95 \mathrm{~mm}$ \\
\hline Outlet radius & $R_{2}$ & $75.25 \mathrm{~mm}$ & outlet radius & $R_{6}$ & $50 \mathrm{~mm}$ \\
\hline Blade height & $b_{i}$ & $12.7 \mathrm{~mm}$ & vane height & $b_{r}$ & $14 \mathrm{~mm}$ \\
\hline Front side chamber height & $h_{f s}$ & $1.85 \mathrm{~mm}$ & - & - & - \\
\hline Back side chamber height & $h_{b s}$ & $2 \mathrm{~mm}$ & - & - & - \\
\hline \multicolumn{3}{|c|}{ Diffuser } & \multicolumn{3}{|c|}{ Design operating point } \\
\hline Number of vanes & $Z_{d}$ & 9 & flow rate & $Q_{\text {des }}$ & $0.0045 \mathrm{~m}^{3} / \mathrm{s}$ \\
\hline Inlet radius & $R_{3}$ & $77.5 \mathrm{~mm}$ & rotating speed & $n_{\text {des }}$ & $1450 \mathrm{rpm}$ \\
\hline Outlet radius & $R_{4}$ & $95 \mathrm{~mm}$ & delivery head & $H_{\text {des }}$ & $7 \mathrm{~m}$ \\
\hline Vane height & $b_{d}$ & $14 \mathrm{~mm}$ & specific speed & $n_{s}$ & 22.6 \\
\hline
\end{tabular}

phase-averaged components $(\bar{u}(x, y, \varphi)$ and $\bar{v}(x, y, \varphi))$ of the absolute velocity $\vec{C}$ in (2) are calculated in (3). The relative velocity based on the relative frame of reference is calculated by (4) by vectorially subtracting the local circumferential velocity from the measured absolute velocity. The turbulence kinetic energy $K$ is calculated in (5), and the turbulence intensity $T_{u}$ is calculated by (6):

$$
\begin{aligned}
u_{i}(x, y, \varphi) & =\bar{u}(x, y, \varphi)+u_{i}^{\prime}(x, y, \varphi), \\
v_{i}(x, y, \varphi) & =\bar{v}(x, y, \varphi)+v_{i}^{\prime}(x, y, \varphi), \quad i=1, \ldots, N, \\
\vec{C}(x, y, \varphi) & =\bar{u}(x, y, \varphi) \vec{i}+\bar{v}(x, y, \varphi) \vec{j}, \\
\bar{u}(x, y, \varphi) & =\frac{1}{N} \sum_{i=1}^{N} u_{i}(x, y, \varphi), \\
\bar{v}(x, y, \varphi) & =\frac{1}{N} \sum_{i=1}^{N} v_{i}(x, y, \varphi), \\
\vec{W} & =\vec{C}-\vec{U}, \\
K(x, y, \varphi) & =\frac{1}{2 N} \sum_{i=1}^{N}\left[u_{i}^{\prime 2}(x, y, \varphi)+v_{i}^{\prime 2}(x, y, \varphi)\right], \\
T u(x, y, \varphi) & =\frac{\sqrt{K(x, y, \varphi)}}{U_{2}} .
\end{aligned}
$$

Here $N$ is the number of measurement at the impeller circumferential position $\varphi$, and $U_{2}$ is the impeller tip speed.

\section{Results}

All the results presented here are limited to the design operating point $Q_{\text {des }}$ and at midspan, that is, at the half blade height. All the velocity components discussed in this chapter are phase-averaged by default.

Figure $4(\mathrm{a})$ presents the phase-averaged relative velocity contours obtained by PIV. The impeller rotation sense is clockwise, and the shown impeller position is defined to be the zero position ( $\varphi=0$ deg, defined in Figure 3$)$ to the predefined diffuser vane, where the impeller trailing edge begins to approach the diffuser leading edge. All other impeller positions are based on this definition.

A positive incidence is found at the impeller leading edge, producing a local region near the suction (concave) side with relatively high relative velocity. The relative velocity on the suction side is bigger than on the pressure (convex) side in the front impeller part. The fact that high-momentum fluid is displaced toward the suction side near the inlet section is in accordance with the potential theory. It also indicates that in the inner part of the passage, the meridional curvature associated with the axial-to-radial entry bend dominates over rotational effects. However, this phenomenon is reversed in the impeller rear part due to the fact that the Coriolis force accumulates strength in large radii and drives the fluid from the suction side to the pressure side. Near the impeller outlet, the jet-wake flow structure is observed, characterized by low relative velocity on the impeller suction side near the trailing edge and relatively high velocity on the corresponding pressure side. In the diffuser region, the stagnation point from PIV is found at the diffuser leading edge deviating slightly to the suction side, producing a negative incidence. There is also a region with small velocities on the diffuser pressure side, and no flow separation can be found. A small wake region with low velocities is also found behind the diffuser vane trailing edge. Figure 4(b) presents the result obtained by LDV measurements at the same impeller position with the same magnitude scale as that in Figure 4(a). Obviously the comparison between PIV and LDV results shows a very good qualitative agreement in the whole overlapping measuring region between them.

Figure 5 shows quantitative comparisons of the relative velocities among the results obtained from PIV, LDV, and $\mathrm{CFD}$ in the overlapping region. $\theta^{*}$ is the normalized circumferential coordinate; $\theta^{*}=0$ and $\theta^{*}=1$ represent the circumferential position on the impeller pressure side (PS) and on the suction side (SS), respectively. For the comparison in the diffuser region, $S^{*}$ denotes the dimensionless distance from the diffuser vane pressure side to the corresponding suction side. $S^{*}=0$ is on the pressure side and $S^{*}=1$ is on the suction side. It can be observed that the agreement between the PIV and LDV results is quite good, especially in the impeller region. The CFD results show very similar 


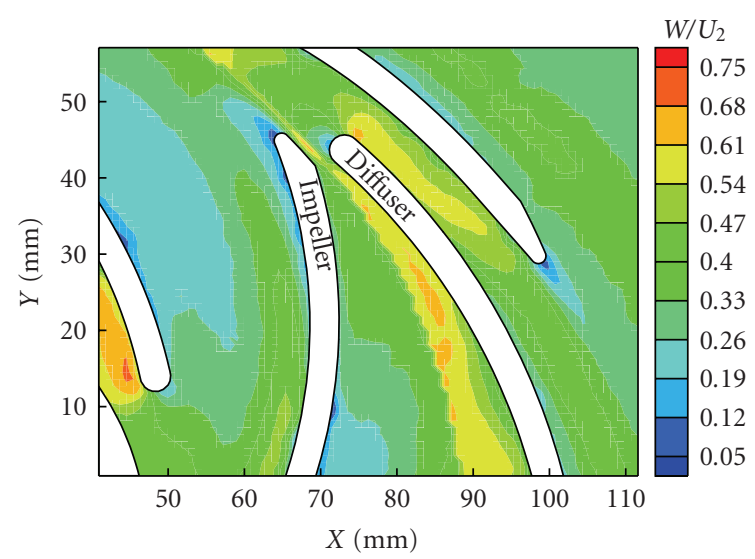

(a) By PIV

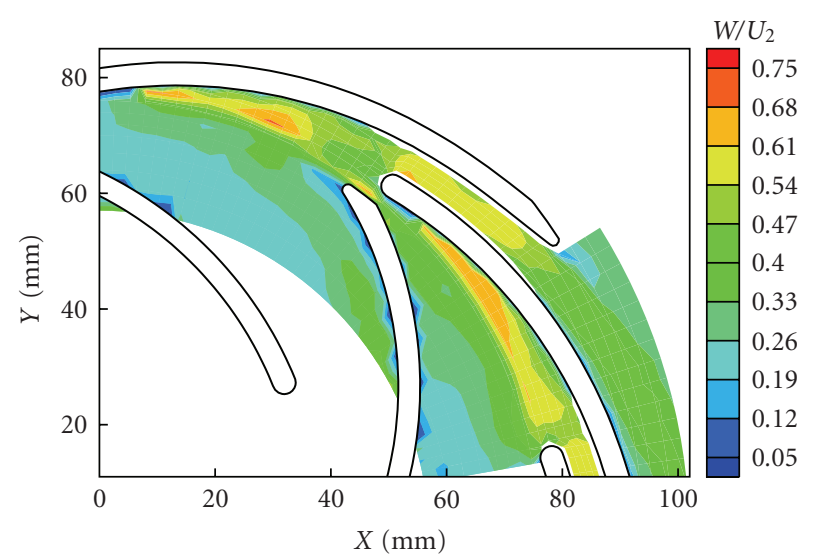

(b) By LDV

FIgURE 4: Phase-averaged relative velocity field at $\varphi=0 \mathrm{deg}$.

trends to the measurement although some discrepancies could be observed. For example, CFD generally slightly underestimates the velocities near the center of the impeller passage and overestimates the velocities near the impeller suction side. However, the relative velocity obtained from the CFD calculation shows an excellent agreement with that by LDV in the radial gap region (at $r / R_{2}=1.01$ in Figure 5(h)). Furthermore, the deficit in the relative velocity near the impeller suction side is very clear at the radial station $r / R_{2}=0.983$ due to the impeller wake. LDV predicts slightly higher magnitude of the relative velocity in the diffuser region, especially at the diffuser outlet throat (Figure $5(\mathrm{j})$ ), where the result by LDV is about $8 \%$ in the middle and $4 \%$ near the blade surfaces higher than that by PIV.

The turbulence intensity obtained by PIV is illustrated in contours in Figure 6(a). High-turbulence regions can be observed on the impeller suction side, behind the impeller trailing edge, around the diffuser leading edge caused by impeller-diffuser interactions, on the diffuser suction side, and behind the diffuser trailing edge. Compared to the PIV result, the LDV result shown in Figure 6(b) predicts similar turbulence trends but with less noise. And the interaction between the impeller and diffuser is very clearly shown by the turbulence distribution in front of the diffuser leading edge and on the suction side of the diffuser vane, which is caused by the impingement between the high-turbulence behind the impeller trailing edge and the diffuser leading edge. It is assumed to be one of the sources of unsteady phenomena in vaned diffuser pumps, which has also been reported by Sinha and Katz [10]. In addition, LDV predicts a wider region extending downstream to the impeller outlet with a higher turbulence level in the impeller channel compared to the PIV result. However, the high-turbulence region around the diffuser leading edge cannot be predicted by LDV due to the limitation of the measuring positions in the LDV.

In the CFD result for a three-dimensional unsteady flow, the turbulence kinetic energy $K(x, y, z, \varphi)$ is contributed by three fluctuating velocity components $\left(u^{\prime}, v^{\prime}\right.$, and $\left.w^{\prime}\right)$. Since only two components are available in the two-dimensional
PIV and LDV measurements, an isotropic assumption is thereby applied in (7) to calculate the turbulence kinetic energy $K(x, y, \varphi)$ considering two components [21], for the comparison with the PIV and LDV results. The turbulence intensity $T u$ is defined in (8) for CFD results, which is the same with that in (6) for LDV and PIV measurements. All CFD results on the turbulence intensity $T_{u}$ presented in this paper are based on this definition:

$$
\begin{aligned}
K(x, y, \varphi) & =\frac{2}{3} K(x, y, z, \varphi), \\
T_{u}(x, y, \varphi) & =\frac{\sqrt{K(x, y, \varphi)}}{U_{2}} .
\end{aligned}
$$

Figure 7 illustrates the turbulence fields from CFD calculations for the same impeller position. Two cases are considered here: with and without consideration of the impeller side chambers. It is found that the turbulence level from CFD is slightly smaller than from the measurements. One reason is that the velocity fluctuations in PIV and LDV are not only caused by turbulence but also caused by some other random errors in the measurement. The turbulence measurement is the combination of the turbulence effect in the flow and the unsteady or fluctuating velocity components due to the relative motion between the measurement volume and the velocity gradients in the flow [22]. Therefore, a different scale is chosen here to better show the turbulence trend for the CFD results. The CFD result with impeller side chambers (Figure 7(a)) predicts similar main features compared with measurements, and nearly all above high-turbulence regions can be also observed in the CFD result. In addition, by comparing the results between with (Figure 7(a)) and without (Figure 7(b)) consideration of side chambers in the simulations, it is confirmed that the high-turbulence region on the impeller suction side, starting near the impeller leading edge, is caused by the leakage flow in the impeller side chambers. In addition, the leakage flow increases the turbulence level in the whole range, especially in the impeller region. 


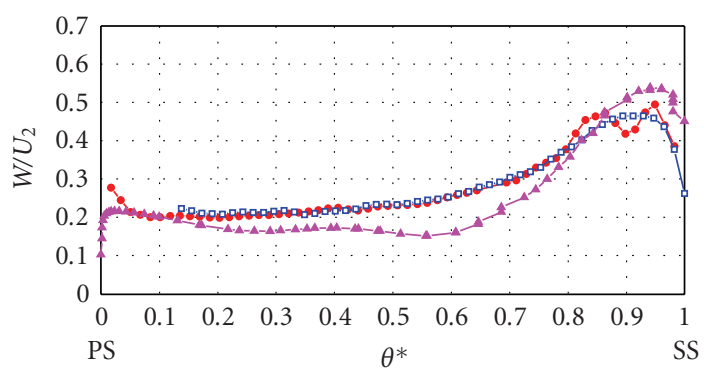

(a) $r / R_{2}=0.757$

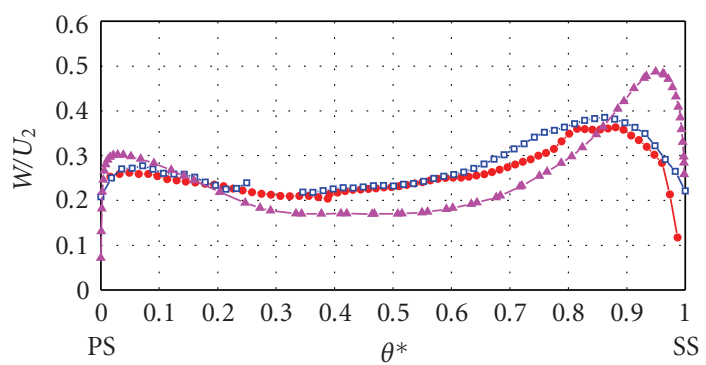

(c) $r / R_{2}=0.850$

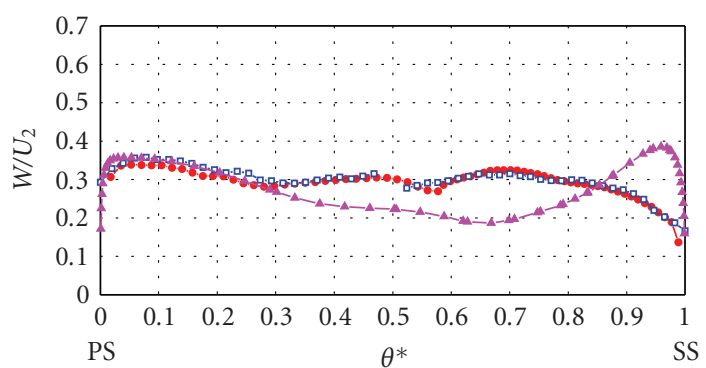

(e) $r / R_{2}=0.930$

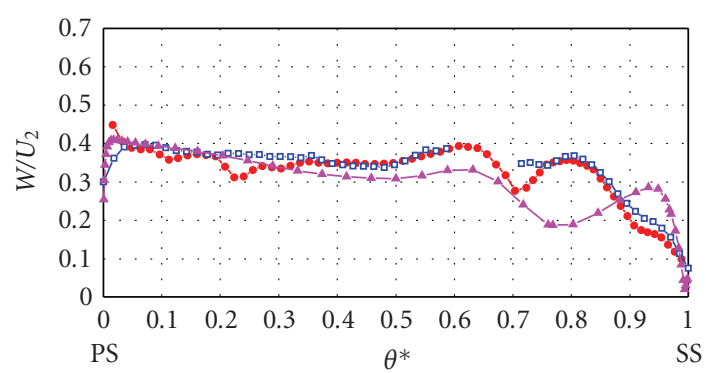

(g) $r / R_{2}=0.983$

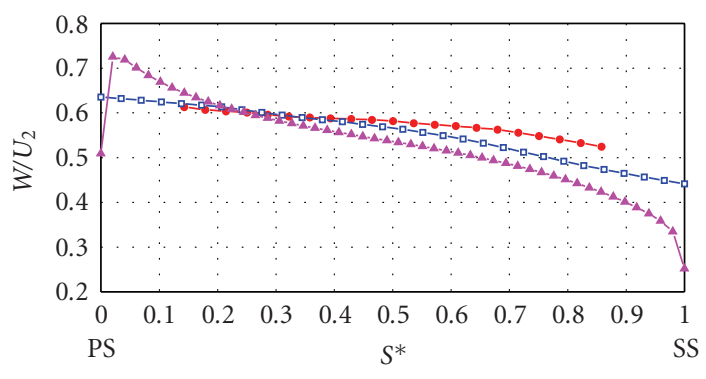

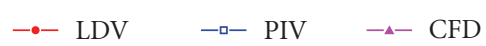

(i) Diffuser inlet throat

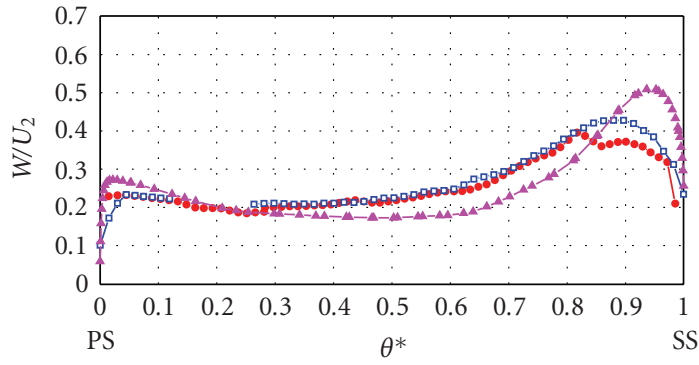

(b) $r / R_{2}=0.811$

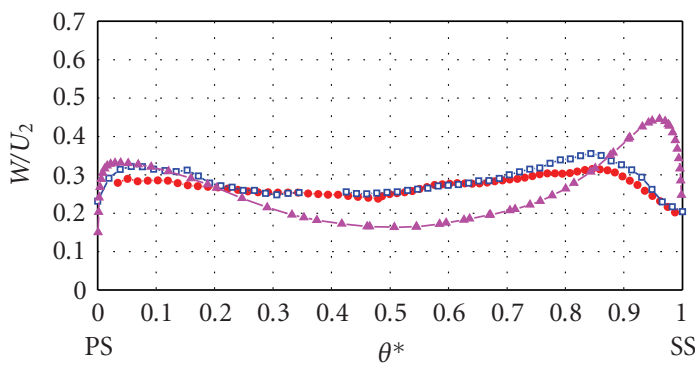

(d) $r / R_{2}=0.890$

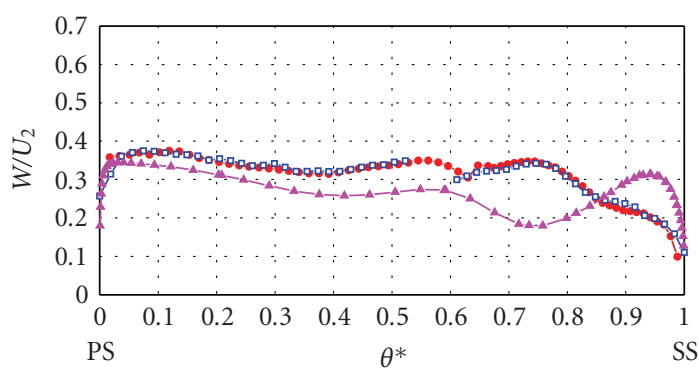

(f) $r / R_{2}=0.957$

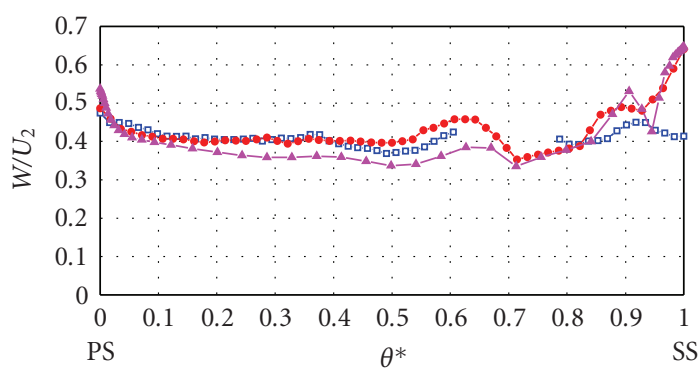

(h) $r / R_{2}=1.010$

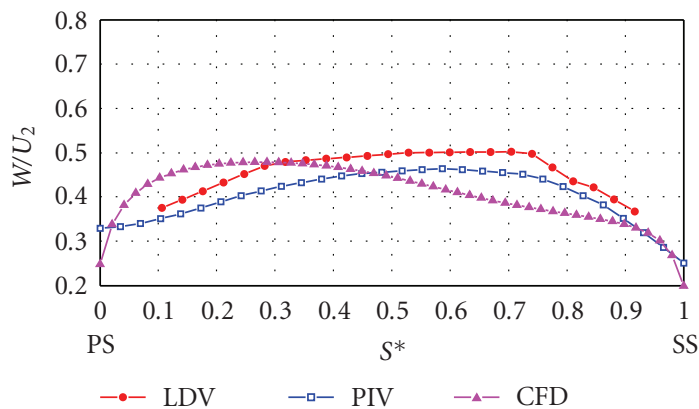

(j) Diffuser outlet throat

FIgURe 5: Comparison of phase-averaged relative velocities at $\varphi=0 \mathrm{deg}$. 


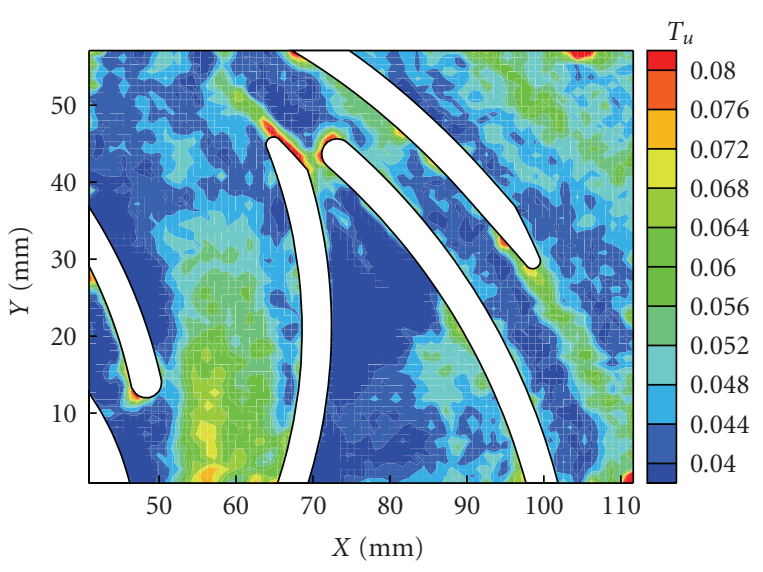

(a) By PIV

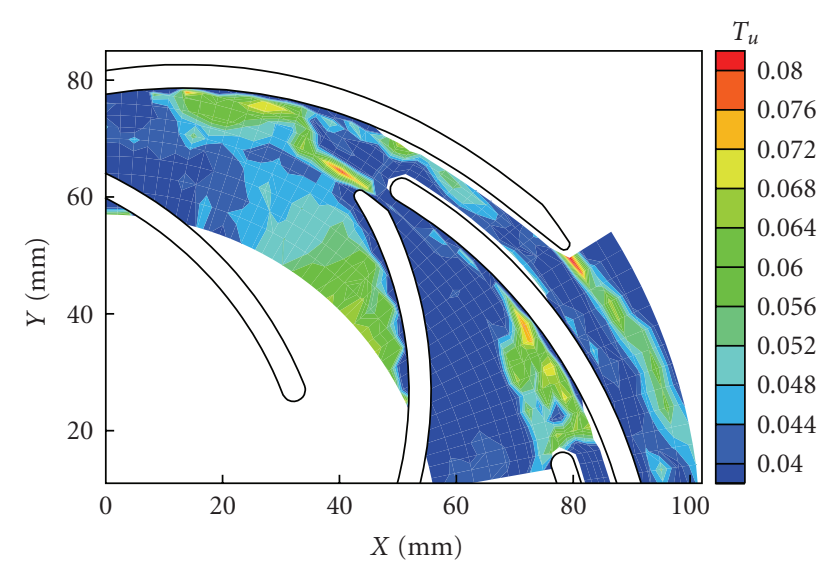

(b) By LDV

FIgURE 6: Turbulence intensity from measurements at $\varphi=0 \mathrm{deg}$.

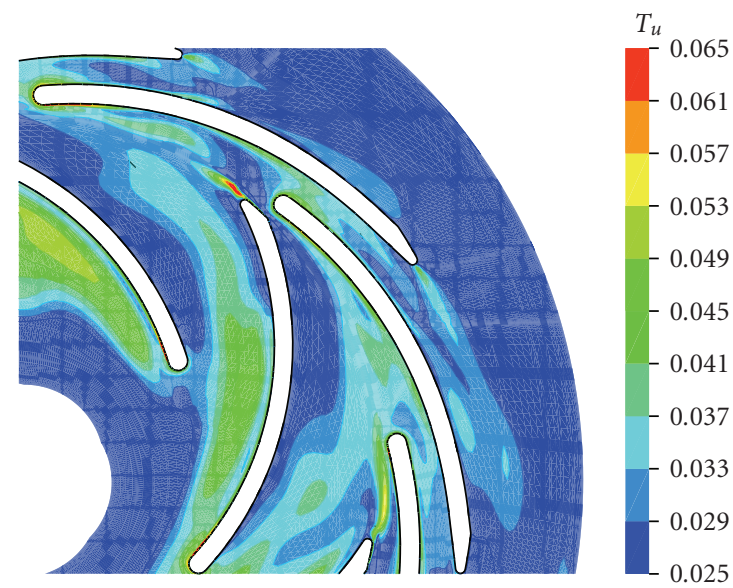

(a) With impeller side chambers

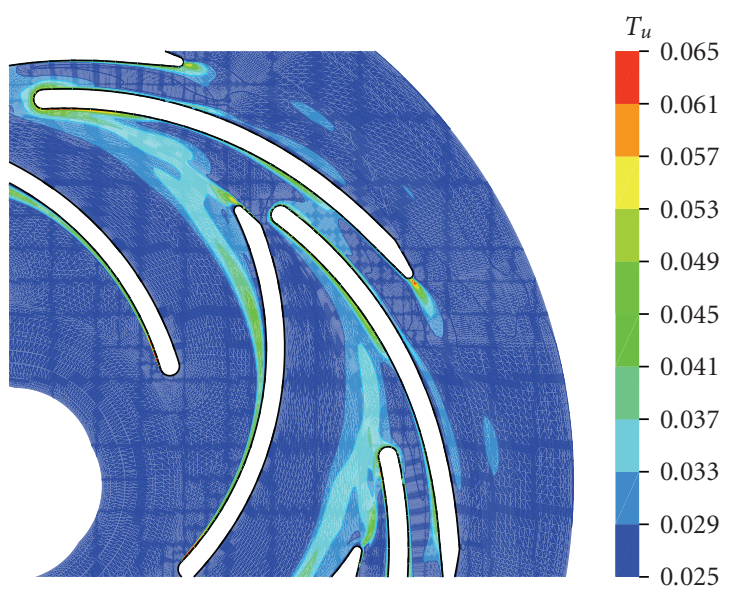

(b) Without impeller side chambers

Figure 7: Turbulence intensity distributions from CFD at $\varphi=0 \mathrm{deg}$.

Figure 8 shows the quantitative comparison of the turbulence intensity fields at the same positions as in Figure 5 among PIV, LDV, and CFD results. One can observe that the agreement between the PIV and LDV results at all impeller positions is generally good. CFD totally underestimates the turbulence but predicts normally similar trends of the turbulence distribution compared with the results by PIV and LDV, except at the diffuser inlet throat. The highest turbulence intensity in the impeller region is observed near the suction side produced by the leakage flow, except for the radial station after $r / R_{2}=0.983$ where the highest turbulence intensity is caused by the high-turbulence region carried by the impeller wake (Figure 6(b)). The impeller wake is very evident at $r / R_{2}=1.01$ (Figure 8(h)), indicated by the highturbulence intensity $(8.2 \%$ by LDV, $7.3 \%$ by PIV and $5.8 \%$ by CFD, based on $U_{2}$ ) very near the impeller suction side. In the diffuser region, PIV predicts a slightly higher magnitude of turbulence than LDV at the inlet and outlet throats. A possible reason for this is due to the reflection on the diffuser vanes in the PIV measurement, since the diffuser channel is very narrow. The effect from the reflection is not negligible to the velocity field, which introduces some other uncertainties in the velocity measurement, and the uncertainties are included in the calculation of the turbulence intensity in the data postprocessing. Both PIV and LDV predict higher Tu near the suction side than near the pressure side at the inlet throat of the diffuser. However, CFD gives a reverse trend. At the diffuser outlet throat (Figure 8(j)), the high-turbulence (about $7 \%$ ) near the suction side is caused by the diffuser wake in the measurement results, and CFD again fails to capture this.

The unsteady interaction is the strongest in the radial gap region between the impeller and the diffuser. Figure 9 shows comparisons of radial and circumferential velocity distributions among the PIV, LDV, and PIV results in the gap region. The comparison of absolute radial component $C_{r}$ (Figures $9(\mathrm{a})$ and $9(\mathrm{~b})$ ) shows a very good agreement between LDV and CFD results, although CFD predicts generally slightly higher values than LDV. However, PIV presents a similar tendency but with a big discrepancy to 


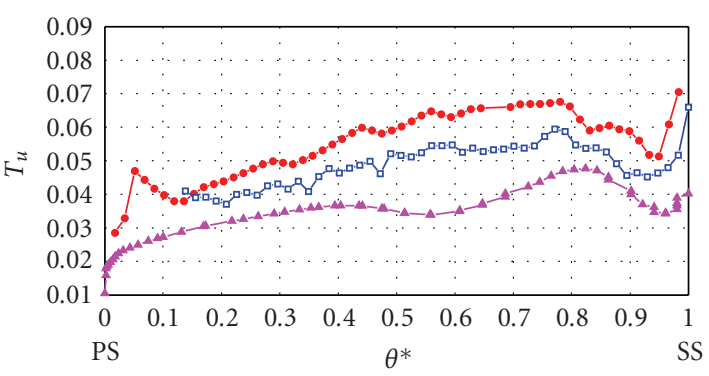

(a) $r / R_{2}=0.757$

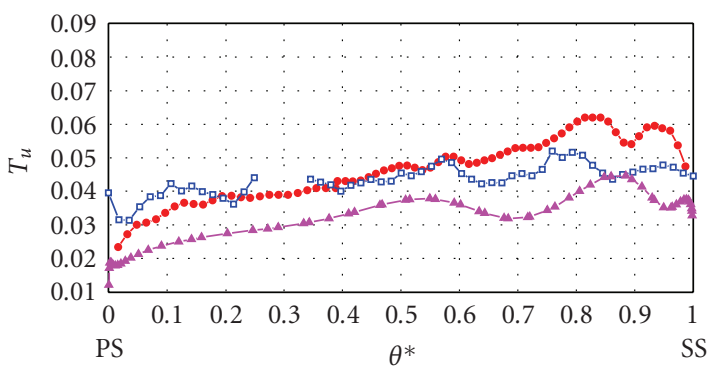

(c) $r / R_{2}=0.850$

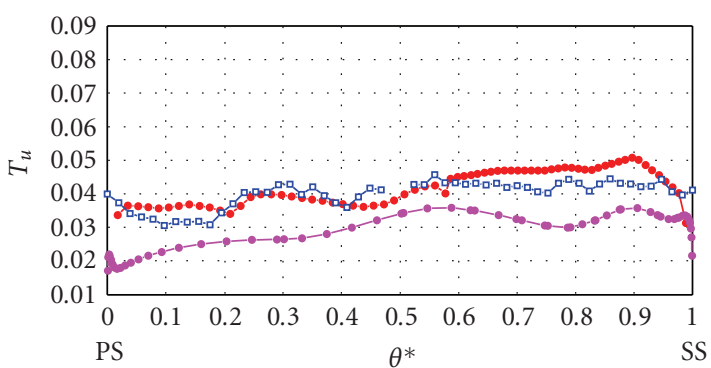

(e) $r / R_{2}=0.930$

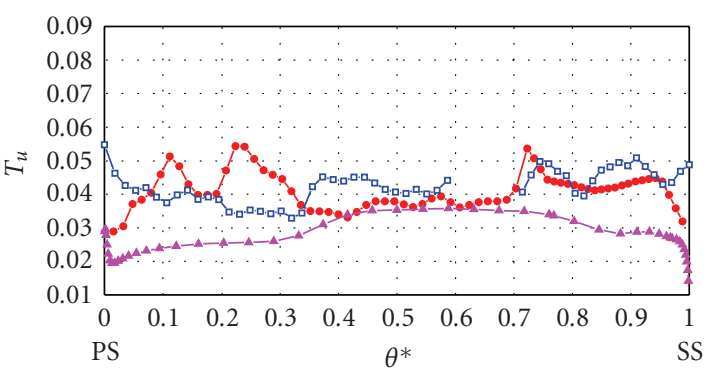

(g) $r / R_{2}=0.983$

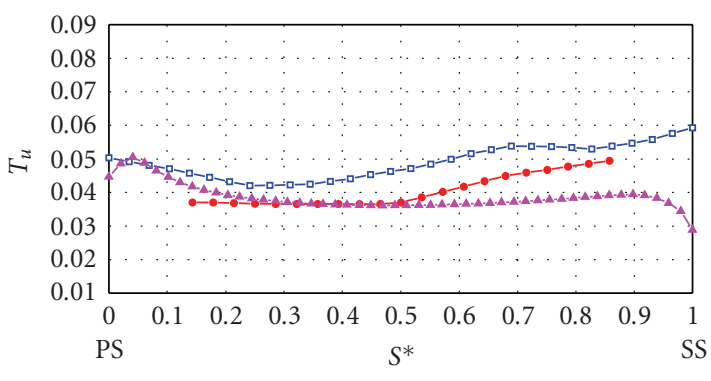

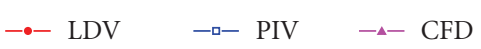

(i) Diffuser inlet throat

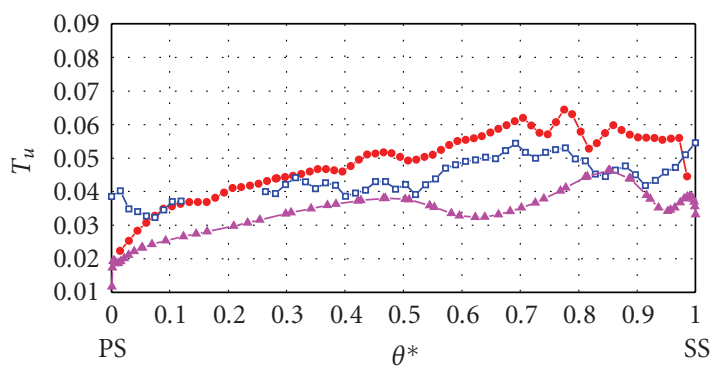

(b) $r / R_{2}=0.811$

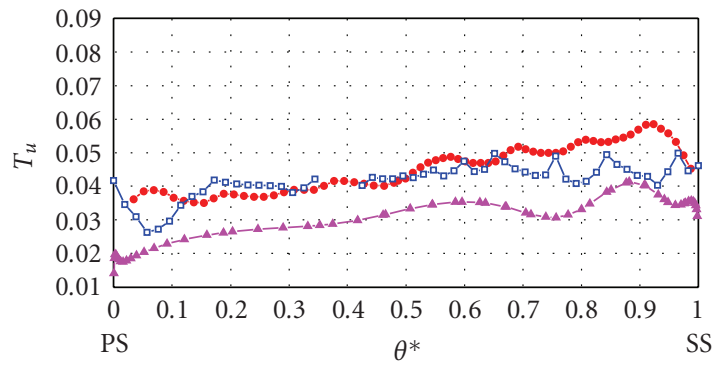

(d) $r / R_{2}=0.890$

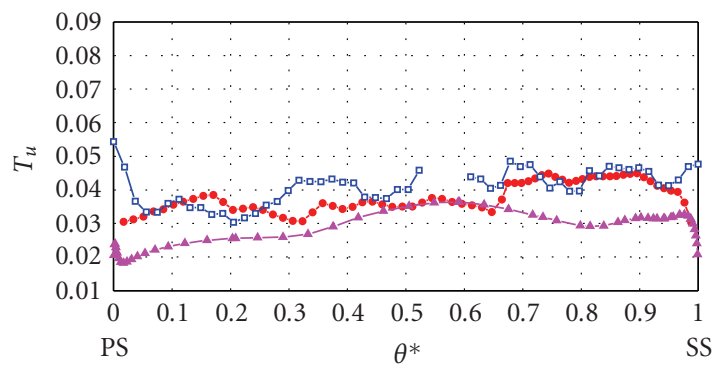

(f) $r / R_{2}=0.957$

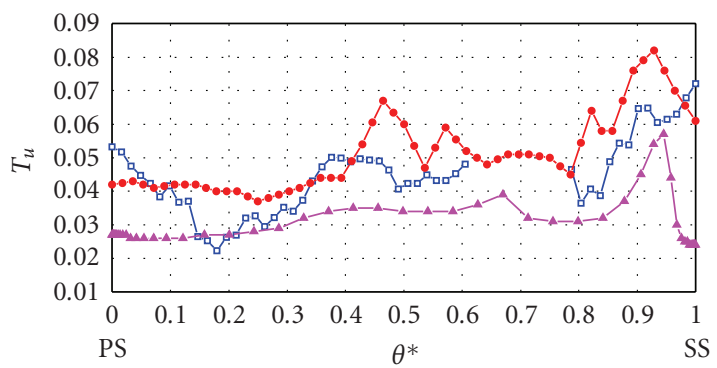

(h) $r / R_{2}=1.010$

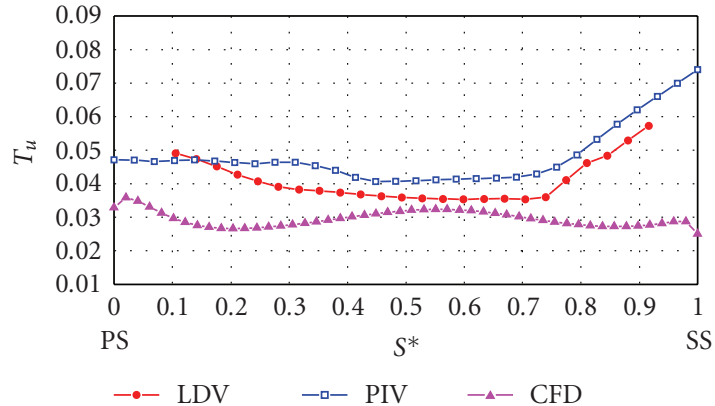

(j) Diffuser outlet throat

Figure 8: Comparison of turbulence intensities at $\varphi=0 \mathrm{deg}$. 


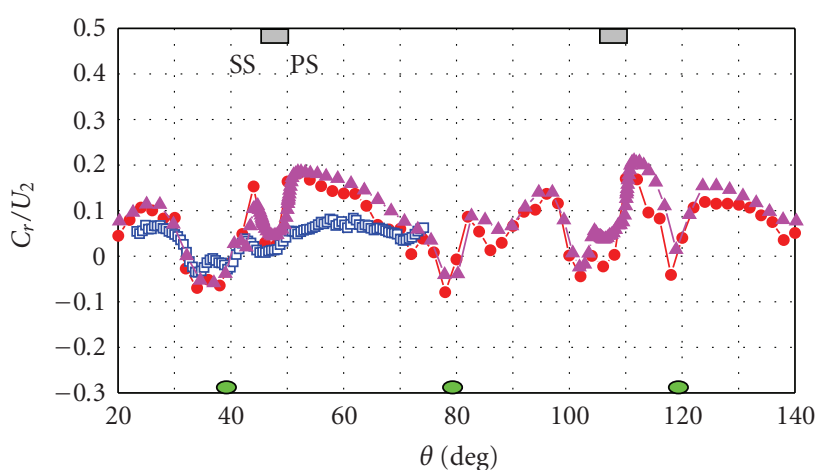

(a) $C_{r}$ at $\varphi=-10 \mathrm{deg}$

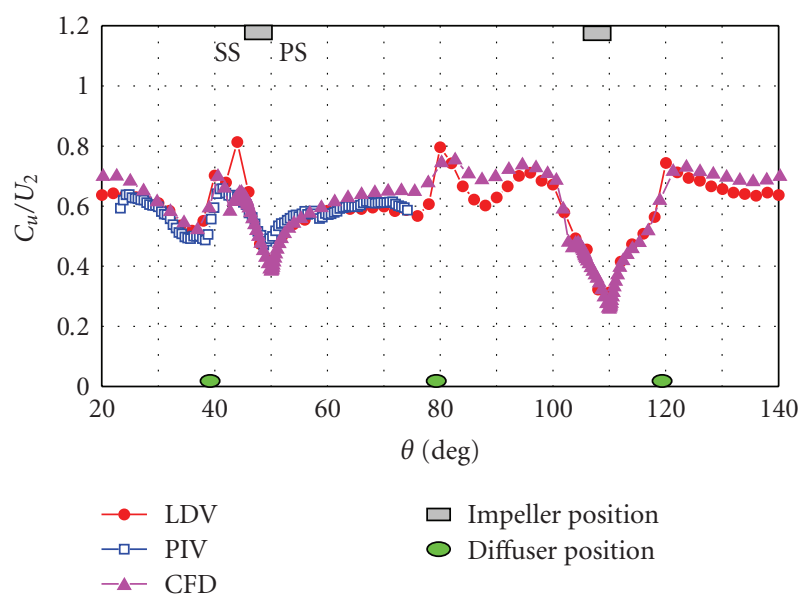

(c) $C_{u}$ at $\varphi=-10 \mathrm{deg}$

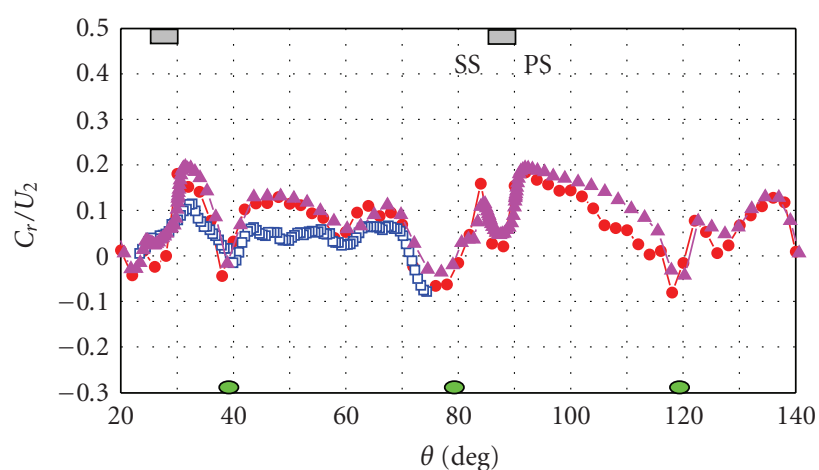

(b) $C_{r}$ at $\varphi=10 \mathrm{deg}$

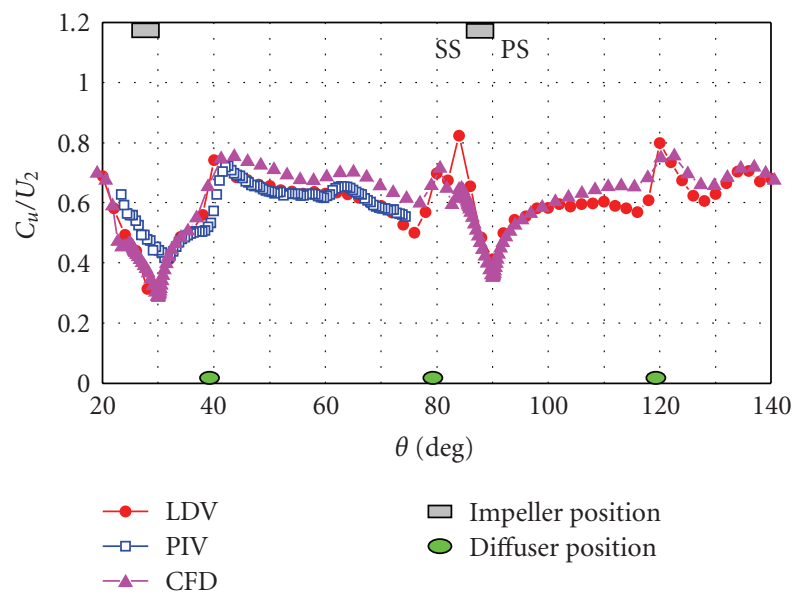

(d) $C_{u}$ at $\varphi=10 \mathrm{deg}$

FIgURE 9: Comparison of velocity components in the radial gap, $r / R_{2}=1.01$.

LDV. For example, both LDV and CFD predict a local high $C_{r}$ region near the pressure side; PIV does it too but with much smaller magnitude. The biggest discrepancy is observed in that region. This phenomenon could be the fact that the reflection in the PIV measurement is very big near the gap region, and the data postprocessing utilizes a moving-average method to generate velocity vectors. The distribution of the radial velocity presents a series of peaks and valleys. The potential effect induced by the diffuser vanes to the impeller outlet flow is very clear: a local minimum of radial velocity could be observed in the vicinity of each diffuser leading edge, and the value is negative indicating local reverse flow. The radial velocity component reaches a peak near the pressure side of the impeller passage, and it decreases to a local minimum in the wake region on the suction side of the passage, where local reverse flow can also be observed which are indicated by the negative radial velocity. For the circumferential velocity component profiles shown in Figures 9(c) and 9(d), PIV and CFD give the same result. CFD again predicts quite a similar result with LDV and PIV with slightly higher amplitude, which is responsible for the slightly higher delivery head in the CFD calculation than that by the measurement in Figure 2. $C_{u}$ attains a valley near the pressure side of the impeller passage, and the peak-to-peak difference is about $0.45 U_{2}$.
The gradient of the circumferential velocity is very big in the region which is faced directly by the impeller trailing edge. Each leading edge of the diffuser vane produces a local high circumferential velocity, except when the impeller trailing edge and the diffuser leading edge are circumferentially aligned. Therefore, the velocity components in the radial gap region strongly depend on the impeller relative position to the diffuser, and the impeller rotation provides different inlet flow conditions for the downstream diffuser.

\section{Conclusions}

In this paper, the velocity and turbulence fields obtained from CFD, PIV, and LDV are compared both qualitatively and quantitatively at the design operating point. The following conclusions can be drawn.

(1) The jet-wake flow structure is observed near the impeller outlet, which is characterized by high relative velocity with low turbulence on the pressure side and low relative velocity and high-turbulence on the suction side.

(2) The high-turbulence regions are observed on the impeller suction side due to the leakage flow in the front side chamber, behind the impeller trailing edge 
due to the impeller wake, around the diffuser leading edge, on the diffuser suction side, and behind the diffuser trailing edge caused by the diffuser wake. The turbulence level caused by the leakage flow in the impeller side chambers is nearly in the same level with the one in the impeller wake (about $8 \%$ by LDV, based on $U_{2}$ ). The turbulence on the impeller pressure side is about $2 \%-3 \%$ by the measurement, which is smaller than on that the impeller suction side. In addition, the turbulence intensity in the diffuser region is slightly higher predicted by PIV than that determined by LDV.

(3) In the radial gap region near the diffuser inlet, the distribution of the absolute radial component $C_{r}$ by the PIV measurement is smoother than that by the LDV measurement. The agreement of $C_{u}$ is better than that of $C_{r}$. The velocity components in the gap region strongly depend on the impeller relative position to the diffuser, and the impeller rotation provides different inlet flow conditions for the downstream diffuser.

(4) PIV and LDV give nearly the same phase-averaged velocity fields. In the blade region, CFD predicts similar velocity profiles as PIV and LDV although some discrepancies appear near the impeller suction side. However, the agreement in the radial gap region becomes much better even for the velocity components. The turbulence field from LDV is much clearer and better than that from PIV due to the reflection from solid surfaces in the PIV measurement. CFD underestimates the turbulence level in the whole region compared with PIV and LDV but gives the same trend.

\section{Nomenclature}

$\theta: \quad$ Circumferential coordinate

$\theta^{*}$ : Normalized circumferential coordinate

$\varphi$ : Impeller circumferential position

C: Absolute velocity

$C_{r}$ : Absolute radial velocity

$C_{u}$ : Absolute circumferential velocity

$H$ : Delivery head

$K$ : Turbulence kinetic energy

$n$ : $\quad$ Rotating speed

Q: Flow rate

$R, r$ : Radius

$S^{*}$ : Dimensionless distance

$T_{u}$ : Turbulence intensity

$U$ : Circumferential velocity

$u$ : Absolute velocity component in $X$ direction

$v$ : Absolute velocity component in $Y$ direction

$W$ : Relative velocity

w: Absolute velocity component in $Z$ direction.

\section{Abbreviations}

PS: Pressure side

SS: Suction side.

\section{Superscripts}
': Fluctuating
-: Averaged.

\section{Subscripts}

1: Impeller inlet

2: Impeller outlet

3: Diffuser inlet

4: Diffuser outlet

d: Diffuser

i: Impeller.

\section{References}

[1] N. Arndt, A. J. Acosta, C. E. Brennen, and T. K. Caughey, "Experimental investigation of rotor-stator interaction in a centrifugal pump with several vaned diffusers," Journal of Turbomachinery, vol. 112, no. 1, pp. 98-108, 1990.

[2] P. F. Bert, J. F. Combes, and J. L. Kueny, "Unsteady flow calculation in a centrifugal pump using a finite element method," in Proceeding of the 18th IAHR Symposium on Hydraulic Machinery and Cavitation, Valencia, Spain, 1996.

[3] L. He and K. Sato, "Numerical solution of incompressible unsteady flows in turbomachinery," Journal of Fluids Engineering, vol. 123, no. 3, pp. 680-685, 2001.

[4] G. Ardizzon and G. Pavesi, "Analysis of unsteady impeller diffuser interaction in a centrifugal pump," in Proceeding of the 22nd IAHR Symposium on Hydraulic Machinery and Systems, Stockholm, Sweden, 2004.

[5] H. Wang and H. Tsukamoto, "Fundamental analysis on rotorstator interaction in a diffuser pump by vortex method," Journal of Fluids Engineering, vol. 123, no. 4, pp. 737-747, 2001.

[6] H. Wang and H. Tsukamoto, "Experimental and numerical study of unsteady flow in a diffuser pump at off-design conditions," Journal of Fluids Engineering, vol. 125, no. 5, pp. 767-778, 2003.

[7] K. W. Cheah, T. S. Lee, S. H. Winoto, and Z. M. Zhao, "Numerical flow simulation in a centrifugal pump at design and off-design conditions," International Journal of Rotating Machinery, vol. 2007, 8 pages, 2007.

[8] M. Raffel, C. Willert, and J. Kompenhans, Particle Image Velocimetry -A Practical Guide, Springer, New York, NY, USA, 1998.

[9] O. Akin and D. Rockwell, "Flow structure in a radial flow pumping system using high-image-density particle image velocimetry," Journal of Fluids Engineering, vol. 116, no. 3, pp. 538-544, 1994.

[10] M. Sinha and J. Katz, "Quantitative visualization of the flow in a centrifugal pump with diffuser vanes-part 1: on flow structures and turbulence," Journal of Fluids Engineering, vol. 122, no. 1, pp. 97-107, 2000.

[11] G. Wuibaut, P. Dupont, G. Caignaert, and G. Bois, "Rotor stator interactions in a vaned diffuser radial flow pump," in Proceeding of the 22nd IAHR Symposium on Hydraulic Machinery and Systems, Stockholm, Sweden, June-July 2004.

[12] G. Wuibaut, G. Bois, P. Dupont, G. Caignaert, and M. Stanislas, "PIV measurements in the impeller and the vaneless diffuser of a radial flow pump in design and off-design 
operating conditions," Journal of Fluids Engineering, vol. 124, no. 3, pp. 791-797, 2002.

[13] J. Feng, F.-K. Benra, and H. J. Dohmen, "Qualitative comparison of unsteady flow between numerical and experimental results in a radial diffuser pump," Journal of Visualization, vol. 10, no. 4, pp. 349-357, 2007.

[14] J. Feng, F.-K. Benra, and H. J. Dohmen, "Unsteady flow visualization at part-load conditions of a radial diffuser pump: by PIV and CFD," Journal of Visualization, vol. 12, no. 1, pp. 65-72, 2009.

[15] A.-R. Akhras, M. El Hajem, R. Morel, and J.-Y. Champagne, "Internal flow investigation of a centrifugal pump at the design point," in Proceeding of the 20th IAHR Symposium on Hydraulic Machinery and Systems, Charlotte, NC, USA, August 2000.

[16] M. El Hajem, A. Akhras, J. Y. Champagne, and R. Morel, "Rotor-stator interaction in a centrifugal pump equipped with a vaned diffuser," in Proceedings of the 4th European Conference on Turbomachinery, Fluid Dynamics and Thermodynamics, Firenze, Italy, March 2001.

[17] G. Pintrand, G. Caignaert, G. Bois, and L. Gros, "Analysis of unsteady flows in a vaned diffuser radial flow pump," in Proceeding of the 21st IAHR Symposium on Hydraulic Machinery and Systems, Lausanne, France, September 2002.

[18] A. Akhras, M. E. Hajem, J. Y. Champagne, and R. Morel, "The flow rate influence on the interaction of a radial pump impeller and the diffuser," International Journal of Rotating Machinery, vol. 10, pp. 309-317, 2004.

[19] F. R. Menter, "Two-equation eddy-viscosity turbulence models for engineering applications," AIAA Journal, vol. 32, no. 8, pp. 1598-1605, 1994.

[20] J. Feng, F.-K. Benra, and H. J. Dohmen, "Numerical investigation on pressure fluctuations for different configurations of vaned diffuser pumps," International Journal of Rotating Machinery, vol. 2007, 10 pages, 2007.

[21] F. Soranna, Y.-C. Chow, O. Uzol, and J. Katz, "The effect of inlet guide vanes wake impingement on the flow structure and turbulence around a rotor blade," Journal of Turbomachinery, vol. 128, no. 1, pp. 82-95, 2006.

[22] N. H. Hesse and J. H. G. Howard, "Experimental investigation of blade loading effects at design flow in rotating passages of centrifugal impellers," Journal of Fluids Engineering, vol. 121, no. 4, pp. 813-823, 1999. 

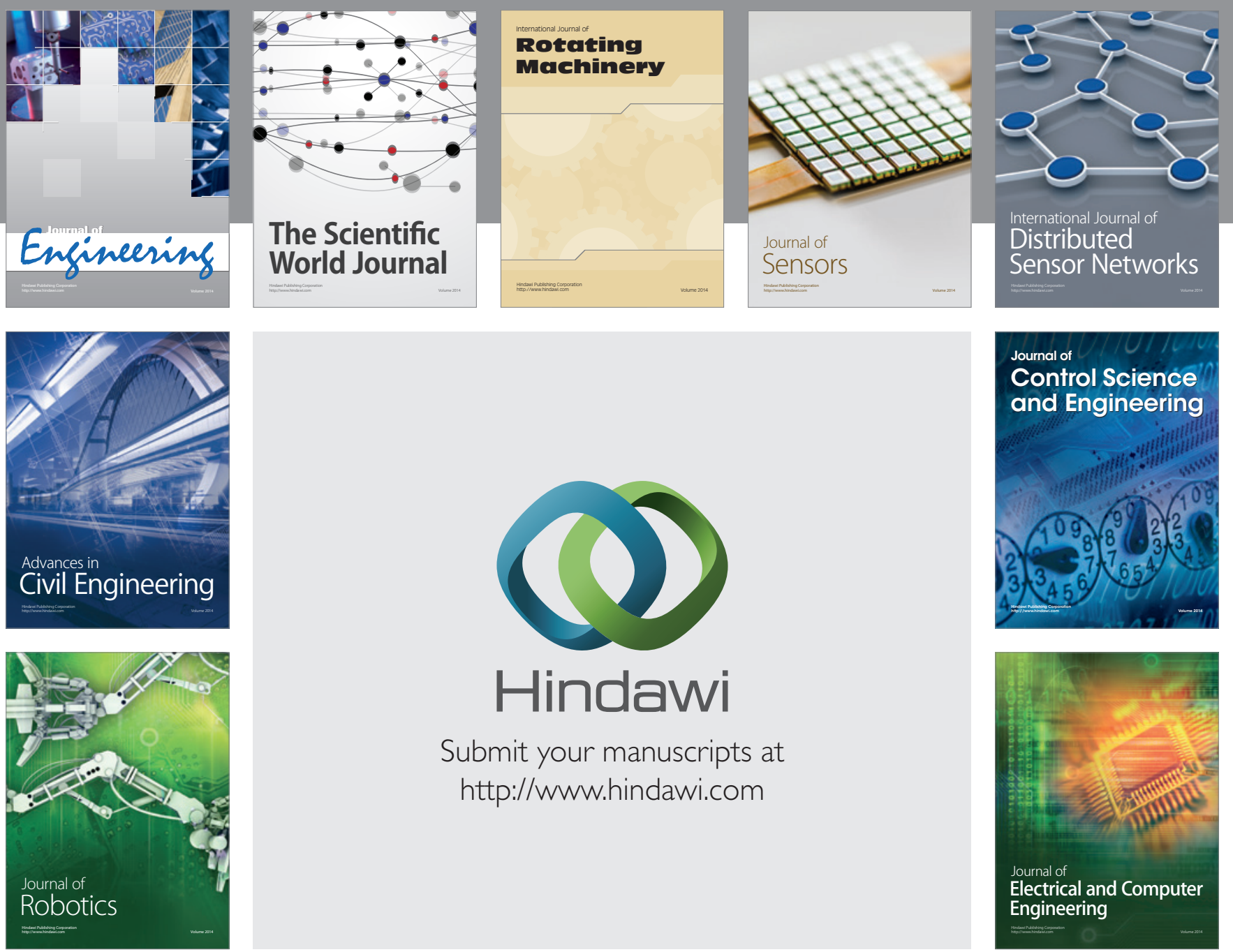

Submit your manuscripts at

http://www.hindawi.com
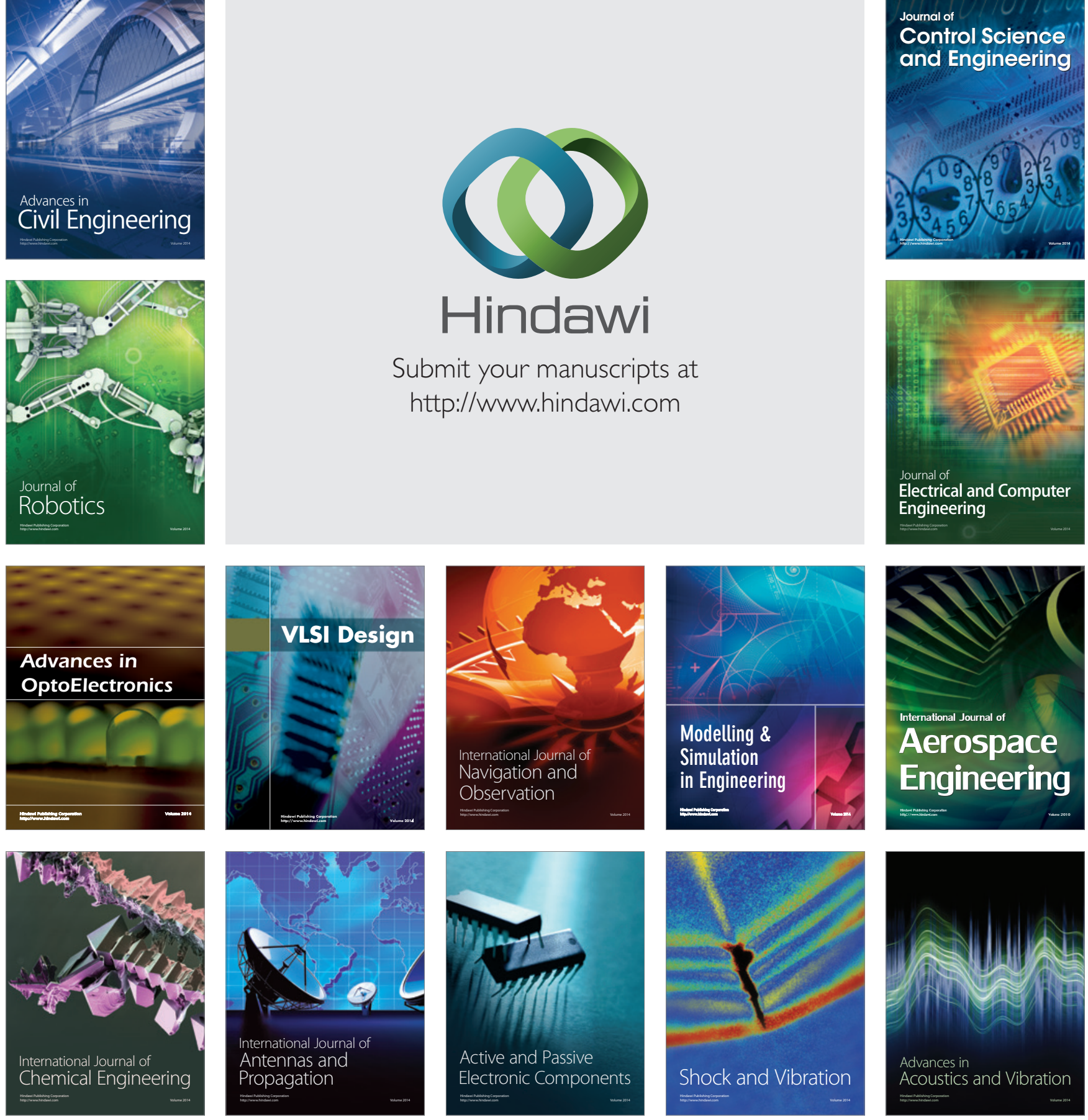